\title{
A reusable laser wrapped graphene-Ag array based SERS sensor for trace detection of genomic DNA methylation
}

Lei Ouyang, ${ }^{\dagger, \S}$ Yaowu Hu, ${ }^{\psi}$ Lihua Zhu, ${ }^{\dagger *}$, Gary J. Cheng, ${ }^{\psi}$ Joseph Irudayaraj ${ }^{\S} *$

$\uparrow$ School of Chemistry and Chemical Engineering, Huazhong University of Science and Technology, Wuhan 430074, P. R. China

$\S$ Department of Agriculture \& Biological Engineering, Bindley Bioscience Center, Purdue Center for Cancer Research, Purdue University, West Lafayette, Indiana, USA 47907

$\psi$ School of Industrial Engineering, Purdue University, West Lafayette, Indiana, USA 47907

\begin{abstract}
Methylation is an important epigenetic DNA modification that governs gene expression. The genomic level of methylated DNA and its derivatives may serve as important indicators for the initiation and progression of cancers among other diseases. In this effort we propose a new laser wrapped graphene-Ag array as a highly sensitive Surface-enhanced Raman spectroscopy (SERS) sensor for the detection of methylated DNA (5-methylcytosine, $5 \mathrm{mC}$ ) and its oxidation derivatives namely 5-hydroxymethylcytosine (5-hmC) and 5-carboxylcytosine (5-caC). Excellent sensitivity and reproducibility were achieved with the laser wrapped graphene-Ag array as a substrate, with the graphene layer acting as an enhancer of the SERS signal due to the effective coupling of the electromagnetic field. In summary, fast (less than $60 \mathrm{~min}$ ) and sensitive (at a limit of detection 0.2

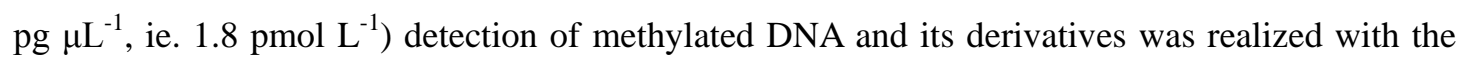
ability to distinguish methylation levels from a mixture at $0.1 \%$. The sensitive and accurate detection in DNA extracted from cells was also accomplished. Furthermore our graphene wrapped approach circumvents the direct interaction between Ag array and the analytes, thus improving the reusability of the SERS substrate even after five cycles of use.
\end{abstract}

Key words: DNA methylation, SERS, laser wrapping, graphene-Ag array.

\section{Introduction}

As one of the most studied epigenetic alterations, DNA methylation (modification of cytosine to 5-methylcytosine, $5 \mathrm{mC}$ ) has drawn much attention because of its important role in numerous cellular processes including embryonic development, genomic imprinting, cellular differentiation. (Ito et al. 2011; Jones and Taylor 1980) Enzymatically mediated epigenetic derivatives of 5mC to 5-hydroxymethylcytosine (5hmC), to 5-formylcytosine (5fC) and 5-carboxylcytosine (5caC) as

The authors declare no competing financial interest.

* Corresponding author. Professor Lihua Zhu Tel: +8618971074688. E-mail: 1hzhu63@ hust.edu.cn; Professor Joseph Irudayaraj Tel: +17654940388. Email: josephi@purdue.edu 
intermediates in the demethylation pathway have also been shown to confer unique transcriptional function to genes. (Ito et al. 2011) More recently, researches have shown that these derivatives are important indicators of the initiation and progression of cancers.(Timp and Feinberg 2013) The global levels of $5 \mathrm{mC}$ have been suggested as a prognostic and diagnostic biomarker depicting early neoplasia and solid tumors.(Feinberg and Vogelstein 1983; Gama-Sosa et al. 1983) A decrease in $5 \mathrm{hmC}$ has been implicated in multiple cancers of the lung, colon, and brain.(Baylin and Jones 2011) Since the dynamic balance between DNA methylation and demethylation, its down-stream components such as $5 \mathrm{fC}$ and $5 \mathrm{caC}$ are also implicated in cancers, quantitative analysis of the genome-wide distribution of these DNA epigenetic modifications could serve as important biomarkers in early cancer detection and therapy monitoring.(Song and He 2012, 2013) Monitoring global methylation level is not only a powerful tool to obtain a snap shot of disease development, but also useful for risk assessment of patients with prolonged viral and bacterial infections.(Shanmuganathan et al. 2013) The profiles of global methylation and its delineation could also be used for studying the mechanism of epigenetic changes and its function in aberrant expression patterns and disease.(Laird 2010) Thus sensitive and accurate monitoring of the global methylation level is important for both basic research and clinical applications.

Different strategies have been proposed to quantify global DNA methylation. Such as methylation specific PCR (MSP), quantitative MSP, pyrosequencing, restriction landmark genomic scanning among other.(Shames et al. 2007) Based on the principle of distinguishing methylated and unmethylated DNA, the strategies could be divided into four main approaches, namely direct chemical identification, endonuclease digestion, bisulphite conversion, and affinity enrichment. With chemical separation and identification strategies, methylated DNA and its derivatives could be directly detected with liquid chromatography (LC) or combined with mass spectrometry (MS).(Huang et al. 2016; Le et al. 2011) These methods usually have excellent sensitivity, but the complex procedures and detection time are not conducive for rapid analyses. The strategy for the digestion of genomic DNA with methylation-sensitive restriction enzymes followed by analysis has been frequently used for methylation analysis combined with sequencing approaches.(Cao and Zhang 2012; Oda et al. 2009; Singer-Sam et al. 1990) The main drawback is that these methods are prone to false-positives that result from incomplete digestion.(Laird 2010) Bisulphite conversion was first proposed by Frommer and coworkers.(Frommer et al. 1992) By employing bisulfite treatment, unmethylated cytosine could be converted to uracil while methylated cytosine $(5 \mathrm{mC})$ remains unchanged. The samples are then used with PCR or other conventional procedures for detection. This technique is time-consuming and the incomplete bisulphite conversion could lead to incorrect results.(Taleat et al. 2015) Another method used for the identification of methylated DNA is the affinity enrichment strategy. By employing antibodies or methyl-binding proteins (MBDs) that are specific to methylated DNA as a scaffold for target genomic DNA capture, a particularly powerful tool was suggested for comprehensive profiling of DNA methylation in complex genomes.(Wang et al. 2012a; Yin et al. 2013) Affinity-based methods allow for the rapid and efficient genome-wide assessment of DNA methylation, and are 
more tolerant of DNA impurity and integrity, which are very suitable for genome-wide detection. Among the most commonly used methods based on affinity enrichment, immunoassays have the potential for ease of use, but they are limited in high-throughput analysis, and for clinical diagnosis, the limited sensitivity and extensive procedures have to be overcome.(Chowdhury et al. 2014) In our earlier work, we proposed a biotin-avidin mediated immunoassay strategy for quantitative determination of methylation with excellent sensitivity, but the method is time-consuming and needs improvement.(Chowdhury et al. 2014; Zhou et al. 2015c) Techniques that could benefit from better signal readout after affinity enrichment needs to be developed with faster and easier operation.

Most strategies for signal read out to assess genomic methylation status focus on the time-consuming PCR with fluorescence detection or sequencing techniques.(Bailey et al. 2009; Chowdhury et al. 2016; Herman et al. 1996) With the goal of developing faster and easier method for methylation detection, significant attention needs to be paid toward nanomaterial-based sensing or imaging because of their sensitivity, ease of use and fast signal output, such as electrochemistry (Kato et al. 2011; Wang et al. 2013; Wu et al. 2013), fluorescence resonance energy transfer (FRET) (Feng et al. 2008; Zhang et al. 2014), Dark Field imaging and Surface Plasmon Resonance (SPR) (Umezu et al. 2011; Wang et al. 2015b; Yu et al. 2010). As one of the most promising technology in trace detection, Surface Enhanced Raman Scattering (SERS) has been frequently used in biosciences because of its advantages in sensitivity, facile pre-operation and rapid analysis process. Earlier work in our group has fully realized its potential in accurate detection of DNA.(Sun et al. 2007, 2008) Several past works also shows sequence-specific methylation or demethylation detection yielding excellent results. However, to date, only a few of these have been proposed for genome-wide detection.(Hu and Zhang 2012; Wang et al. 2015a; Wang et al. 2012b) Recently, methylation-specific MBD protein based SERS strategy by affinity enrichment was proposed for methylation detection.(Wang et al. 2016) However, for effective SERS detection of Raman inactive methylated DNA, selective target capture, specific marking, and proper enhancement by the substrate is very important. Here we propose a unique SERS strategy for trace detection of methylated DNA and its derivatives based on methylation-sensitive antibody acting as a scaffold and a special dye SYBR Green I (SG) as Raman tag which is SERS active only when bound to target DNA (Zhou et al. 2015a). In order to further enhancing the marked target, single layer graphene is wrapped onto the self-assembled Ag array as SERS substrate with a laser shock to provide effective transfer of the electromagnetic field from the metal array to the captured DNA. Different from most of the reported graphene-based SERS substrate whose interaction between the covered plasmonic structure and graphene is not very effective, a laser wrapping strategy is proposed for the enhancement of such interaction.(Du et al. 2014; Gao et al. 2014) The laser shock method with a few GPa pressure can help in the seamless nano-shaping of atomic sheets of graphene onto the plasmonic structures to fully utilize the function of both metal array and graphene.(Hu et al. 2015; Lee et al. 2015) At the same time, the stable and pinhole-free shell can protect the inner metal core and prevent the direct 
metal-molecule contact, enabling reusability of the SERS sensor.

Here by making use of the methylation-specific antibody modified AuNPs as the target scaffold to enrich methylation related DNA, and laser wrapped graphene-Ag array as an enhancing substrate, a highly sensitive and rapid SERS-based DNA sensing strategy was proposed for genomic methylation and its derivatives detection including $5 \mathrm{mC}, 5 \mathrm{hmC}$ and also $5 \mathrm{caC}$. A limit of detection (LOD) as low as $0.2 \mathrm{pg} \mathrm{uL}^{-1}\left(1.8 \mathrm{pmol} \mathrm{L}^{-1}\right)$ of $5 \mathrm{mC}$ levels in a mixture as well as real cell samples at $0.1 \%$ utilizing a small amount ( $200 \mathrm{pg}$ genomic DNA) of input DNA was achieved. The developed method is facile and rapid and proposes to be a promising candidate to detect methylated DNA and its derivatives. The SERS based method could be used as an effective strategy to obtain the global methylation level which can serve as an important indicator for cancer staging and therapy assessment. The proposed unique SERS sensor could not only be used for methylation detection, but also be applied in other biomarker analysis such as phosphorylation.

\section{Result and discussion}

With the goal of developing a better strategy for the detection of DNA methylation, an affinity enrichment-based laser wrapped graphene-Ag array enhanced SERS strategy is proposed. The whole strategy of nanostructure fabrication and detection of methylated DNA and its derivatives is shown in Scheme 1. The detailed synthesis and application steps and parameters of the proposed sensor are included in the Supplementary Material. The target DNA is recognized and captured from the global DNA by the specific antibody. After separation by centrifugation and washing, the captured DNA is marked with the Raman tag, SG, which could selectivity bind to dsDNA to yield a sensitive SERS response. Then Au-DNA conjugates are directly dipped onto the graphene wrapped Ag array for further SERS enhancement. No further separation is needed since SG was SERS inactive in solution. After SERS observation, the Au conjugates could be easily washed away and the graphene-Ag substrate is ready for another round of detection. Details of the synthesis steps and related characterization are stated in the following sections.

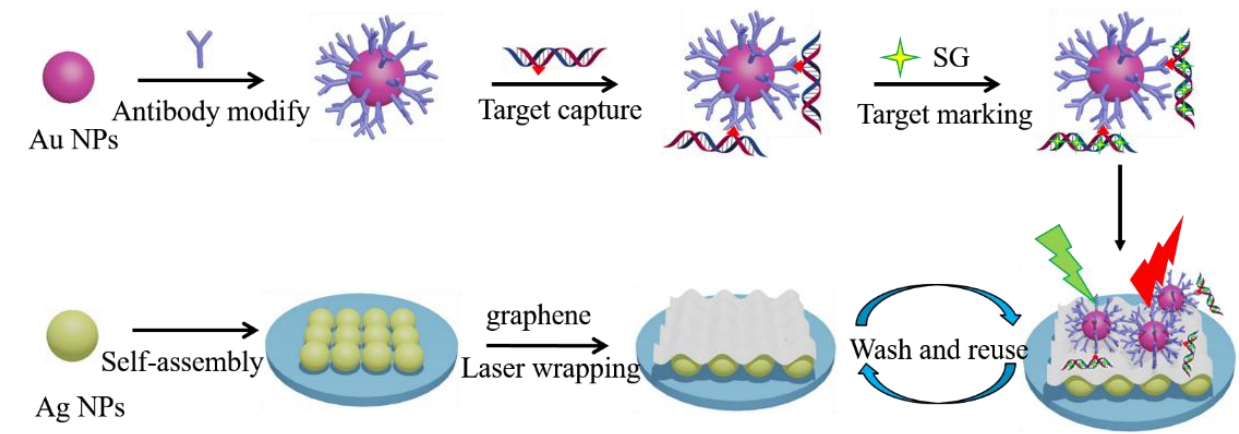

Scheme 1. Illustration of the proposed SERS strategy for detection of methylated DNA and its derivatives. 


\subsection{Antibody modified AuNPs fabrication and characterization}

AuNPs is the most commonly used nanostructure for a variety of targets because of its facile synthesis procedure, distinct optical properties and diverse surface functionalization options.(Zhou et al. 2015b) Here we synthesized AuNPs as the capture scaffold for affinity enrichment of target DNA with specific antibodies. For the AuNPs used in our experiments, the plasmonic peak was observed at $527 \mathrm{~nm}$ (Fig S1b), typical of $35 \mathrm{~nm}$ Au nano-spheres, which matches well with the result from TEM observation (Fig. S1a). The obtained NPs were then mixed with the methylated DNA sensitive antibody. The antibody would attach to the surface of the NPs because of the thiol and amino functionalized groups, to result in a clear red-shift in the plasmonic mode (from 527 $\mathrm{nm}$ to $533.5 \mathrm{~nm}$ ) as observed in the UV-vis spectrum. (Fig. S1b). To block the residual gold surface, casein was used to inhibit the nonspecific binding between Au NPs and the unmethylated DNA. In order to further confirm the successful modification of antibody to the AuNPs surface, Enzyme-linked immunosorbent assay (ELISA) was performed (details of the procedure could be found in the experimental section). Obtained results are shown in Fig. S2, which showed the successful attachment of antibodies to AuNPs endowing the ability for targeting a specific DNA. The modified AuNPs were applied to capture and enrich the target DNA.

\subsection{Labeling of target DNA}

After the target DNA is captured by the antibody modified AuNPs, it would still be difficult to detect the targets by direct SERS observation since the methylated DNA has a weak Raman response. In order to mark the captured DNA with Raman-active probe, we chose a specific molecule, SG which is commonly used as a fluorescence dye for the detection of dsDNA. Despite its excellent performance in fluorescence, it also has excellent SERS signal for DNA detection based on its unique property.(Zhou et al. 2015a) The molecule is SERS active when it binds to the dsDNA, but SERS inactive when it is in solution in the unbound state. As shown in Fig. S3, the SERS response of SG could be enhanced $\sim 15$ times when mixed with dsDNA, while in solution its response is very weak and does not provide pronounced Raman signal. Different from the commonly used Raman tag such as R6G, which has strong background interference, the marker chosen in our study provides a clean background signal which would be very suitable for detecting low levels of target.

\subsection{Self-assembled Ag array formation}

Even though AuNPs have been frequently used as SERS substrate, AgNPs provide greater extinction coefficient and SERS enhancement and more suitable for trace detection in the visible wavelength region.(Garcia-Leis et al. 2013; Li et al. 2013) Here we used citrate reduced AgNPs as 
signal amplifier to further enhance the SERS response of the SG marked target DNA. The obtained AgNPs were monitored by Transmission electron microscope (TEM) as shown in Fig. S4c (size was about 60-80 nm), based on our former experience, the Ag NPs of this size could yield excellent SERS enhancement when excited with a $785 \mathrm{~nm}$ laser.(Ouyang et al. 2015) Instead of directly using the AgNPs in its solution state, we performed an interface self-assembly to obtain uniform Ag array to form "hot spots" for better SERS enhancement. As shown in Fig. S4a, the interfacial self-assembly was obtained by first mixing Ag solution with toluene (toluene: $\mathrm{Ag}$ sol=1:1, v/v), because of the immiscible property, they separated into two phases. Then ethanol (ethanol: $\mathrm{Ag}$ sol =2:3, v/v) was injected into the mixture. After several minutes, the assembled Ag film with metallic luster was formed at the interface between two phases. The assembled Ag array was then transferred onto a round coverslip for further use. The morphology of the Ag array could be found in Fig. S4d, where the AgNPs were closely packed with a narrow gap of 2-3 nm, which could produce SERS "hot spots". Comparison of the SERS performance of AgNPs before and after assembly was shown in Fig. S4b. By using $5 \mathrm{ng} \mu \mathrm{L}^{-1}$ of $5 \mathrm{mC}$ DNA which was marked with 2 $\mu \mathrm{mol} \mathrm{L}{ }^{-1} \mathrm{SG}$ as the SERS probe, an enhancement of $\sim 9.6$ times was observed. More importantly, such uniform self-assembly operation could not only produce significant enhancement, but can also ensure good homogeneity at different positions, which would be better than the sol based substrate with uncontrollable aggregation during SERS detection. Such a two-dimensional structure is also convenient to be wrapped with single layer of graphene suitable for further functionalization.

\subsection{Graphene wrapping with laser shock}

Graphene is a unique two-dimensional material because it could act as an excellent anchor for biomolecules and it is transparent to laser light as well as to the localized electric field, which would be very useful in SERS application. Graphene also supports the plasmon field and its high thermal conductivity could prevent heat localization, when graphene is utilized in sharp plasmonic structures that have intense enhancements and temperature fields.(Xu et al. 2016) Here we made use of a single layer graphene as a further signal enhancing structure. As a first step, we transferred a single layer of graphene to cover onto the Ag nano array (two-dimensional Ag film on the coverslip with a diameter of $\sim 5 \mathrm{~mm}$ ). A rendition of the concept and Scanning Electron microscope (SEM) observation was shown in Fig. $1 \mathrm{~b}$ and e, respectively, where the morphologies were in sharp contrast to those without graphene (a and d). Ultrathin graphene layer on silver nanoarrays provides enhancement to the Raman signal due to effective transfer of the electromagnetic field from the metal core through the shell to the probe molecule.(Xu et al. 2016) At the same time, the stable and pinhole-free shell can protect the inner metal core and prevents the direct metal-molecule contact. We also noticed that by simply covering the Ag array with graphene, the interaction was not effective because of the weak interaction between single layer graphene and AgNPs. Even though the array was covered by graphene, gaps between them still 
existed which prevented effective coupling between two components, because of insufficient transfer of the electromagnetic field. As found in our former work, if we further performed a laser shock on such a covered structure, graphene would be further "wrapped" onto the structure below, leading to a close contact between graphene and the underlying structure.(Gao et al. 2014; Hu et al. 2015) As shown in Fig.1 c and f, after laser shock, the graphene was attached closer to the Ag array, and the shaping effect of the Ag array was more obvious. Such a close contact between graphene and $\mathrm{Ag}$ array is expected to improve the electromagnetic hot spots at the interface of graphene and Ag array. FDTD simulation was performed to compare the electromagnetic field distributions of the Ag array before and after graphene coating, as shown in Fig.1 g, h, and i. Results showed that the maximum electromagnetic field at the junction was enhanced 1.3 times (based on the simulated electromagnetic distribution) when graphene was transferred and further by 1.2 times after graphene wrapping by laser shock. The SERS performance after laser wrapping was monitored and compared to that of a bare Ag array, as shown in Fig $1 \mathrm{k}$ and 1 . The graphene covered Ag array showed 2.4 times more enhancement, and after laser shock, a further 1.5 times strengthening was observed. Such an enhancement is possible due to the increased coupling of the electromagnetic field which matches well with the results from SERS (enhancement factor is proportional to $|\mathrm{E}|^{4}$ ).(Stiles et al. 2008) The electromagnetic field is more confined in a narrower region of the graphene wrapped $\mathrm{Ag}$ array, which leads to a more effective enhancement at the junction between the AgNPs.(Xu et al. 2016) The enhancement factor (EF) of the proposed substrate was also obtained by employing 4-Mercaptobenzoic acid (MBA) as the Raman label, where, the calculated EF value is estimated as $5.89 \times 10^{5}$, further validating the SERS performance of the proposed substrate (detailed calculation is provided in Supplementary Material and Fig. S5). 

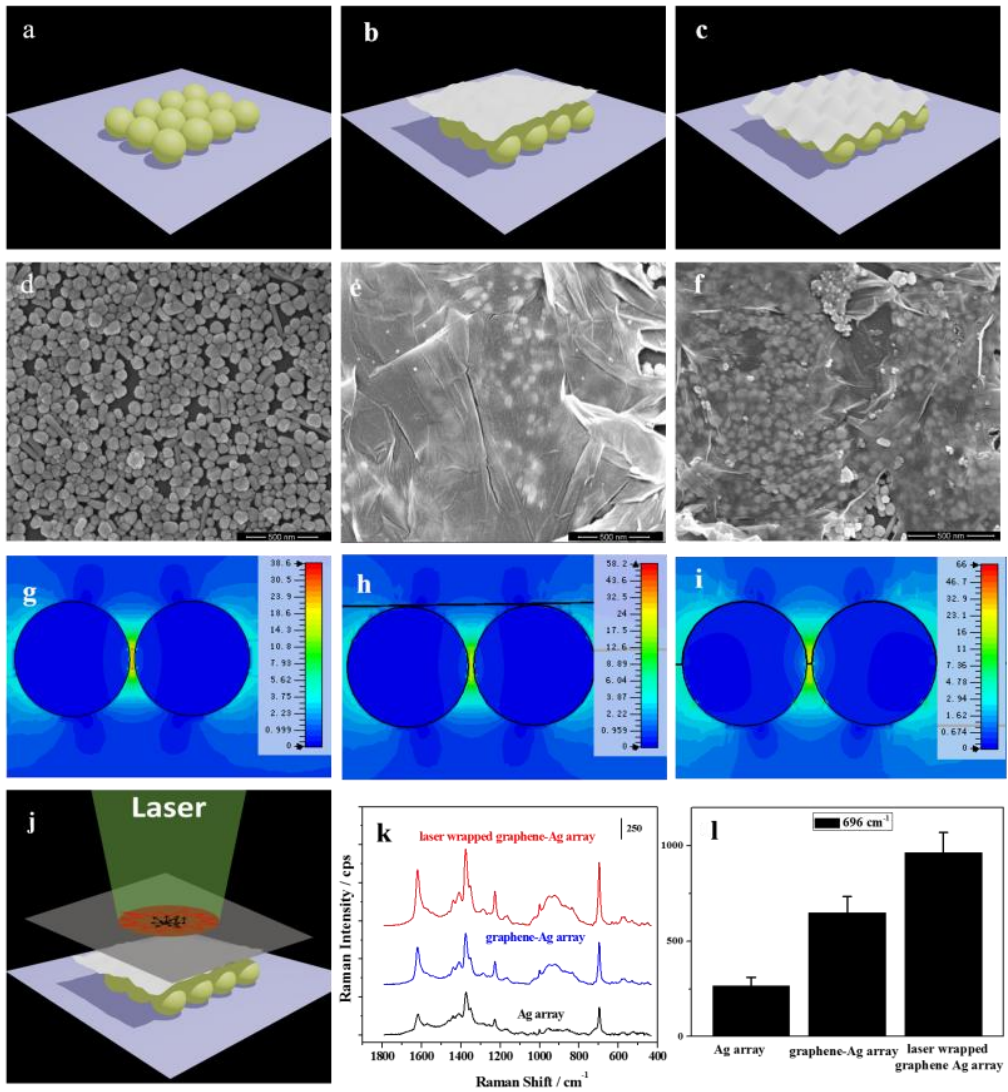

Fig.1 Illustration of the concept and SEM images of Ag array before and after graphene wrapping. a, d Ag array before graphene wrapping; b, e graphene covered Ag array; c, f laser wrapped graphene-Ag array. Simulated electromagnetic field distributions. g AgNPs; h graphene covered Ag array; i graphene wrapped Ag array after laser shock. j Schematic illustration of laser induced wrapping of graphene on the Ag array. k SERS spectrum of SG-DNA with Ag array, graphene covered Ag array and graphene wrapped Ag array as substrate. 1 SERS intensity at $696 \mathrm{~cm}^{-1}$ in $\mathrm{k}$.

\subsection{SERS detection of 5mC DNA in standard, mixture, and real cell samples}

After fabrication of the capture scaffold (antibody modified AuNPs) and the signal enhancer (graphene wrapped Ag array), a strategy for SERS detection of methylation related DNA was proposed as depicted in Scheme 1. The target DNA was first captured by specific antibody modified AuNPs. After centrifugation and washing with water, the interference from nonspecific binding was eliminated. The obtained AuNPs with target DNA was marked with SG, and then dipped onto the graphene-Ag array for SERS observation. Fig. 2a shows the SERS spectra with different dosing concentrations of standard 5mC DNA. An obvious intensity trend was observed with concentration. By choosing the characteristic peak at $696 \mathrm{~cm}^{-1}$ as a representative peak, the LOD was $\sim 0.5 \pm 0.1 \mathrm{pg} \mu \mathrm{L}^{-1}\left(\sim 4.5 \mathrm{pmol} \mathrm{L}^{-1}\right)$. The intensity at this peak was further plotted with respect to their concentrations, as shown in Fig. 2 b. A linear correlation between the peak intensity and the logarithm of the $5 \mathrm{mC}$ DNA concentration over the range of $5 \times 10^{-3}-5 \mathrm{ng}_{\mu} \mathrm{L}^{-1}$ was obtained, 
as shown in the insert of Fig. 2b. These results showed that the performance of the SERS sensor to detect $5 \mathrm{mC}$ DNA was comparable and even lower than the sensitive detection accomplished by our proposed biotin-streptavidin modified ELISA method.(Chowdhury et al. 2014) We should also note that with the developed SERS concept, the detection time is greatly reduced (from 5 hours for ELISA based method to less than 60 minutes).(Chowdhury et al. 2014) Our facile and sensitive approach will provide an excellent prospect for routine and highly sensitive analysis of a large number of samples and an option to be integrated with a portable Raman system.

To validate our proposed method to detect methylation levels in real samples that usually contains a mixture of methylated and unmethylated DNA, artificial mixtures comprising of methylated and unmethylated standard DNA at different ratios were prepared while keeping the total concentration of $5 \mathrm{ng} \mu \mathrm{L}^{-1}$ unchanged (detailed experimental parameter could be found in Supplementary Material). As shown in Fig. $2 c$ and d, the SERS signal increased with an increase in the methylation level, when the methylation level was set at $0 \%$, which means no methylation occurred in the DNA, and nearly no signal was obtained, even though $5 \mathrm{ng} \mu \mathrm{L}^{-1}$ of unmethylated DNA still existed, exhibiting excellent anti-interference ability. It is worth noting that our method can also distinguish methylation level as low as $0.1 \%$, which is more sensitive than former reported methods such as single base extension reaction-based SERS which has a detection limit of $\sim 1 \%$. (Hu and Zhang 2012) Excellent sensitivity is possible due to the specific marker SG and its low background signal. Since no PCR or another extension reaction is required in our method, we expect the result to be more accurate without operation induced false or incorrect readout. We further applied our method for the detection of several real samples. The sample DNA was extracted from a human glioblastoma (GBM) SF767 cell line, the cells were cultured and their DNA were extracted for methylation level analysis with both our proposed SERS method and HPLC/MS/MS method. The detected $5 \mathrm{mC}$ level was about $2.43 \pm 0.15 \%$ with our method, while by HPLC/MS/MS, the methylation level was calculated to be around $2.26 \pm 0.20 \%$. These results clearly show the ability of our method for quantitative analysis of global methylation level.
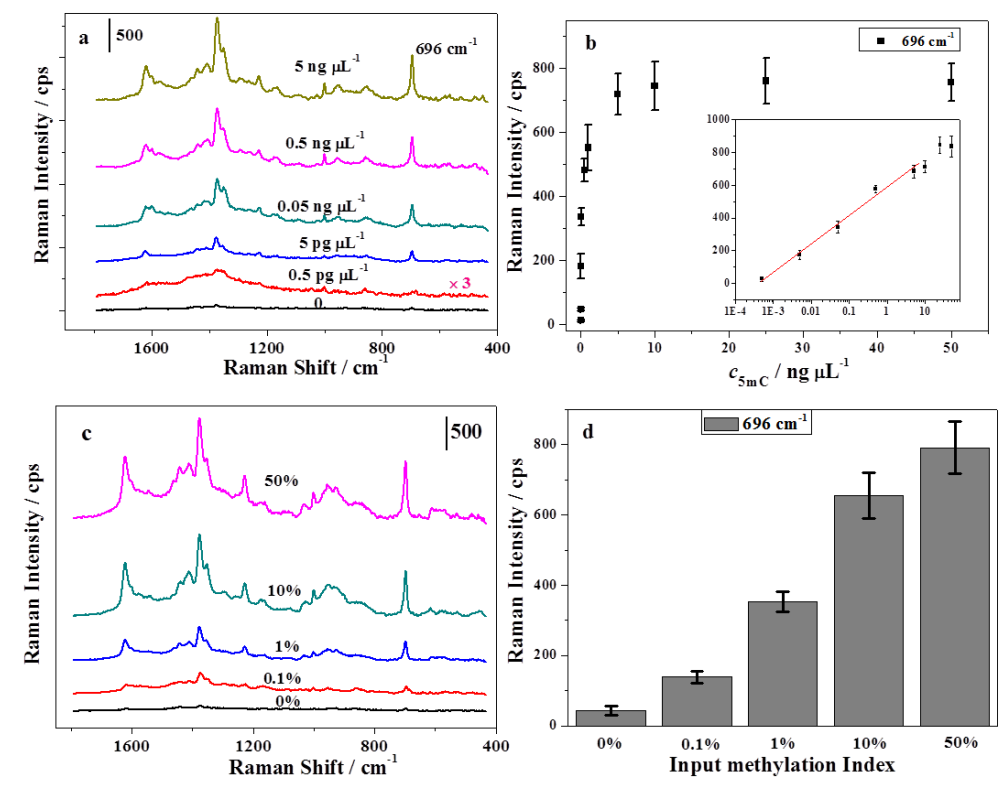
Fig. 2. SERS spectrum of $2 \mu \mathrm{mol} \mathrm{L}{ }^{-1} \mathrm{SG}$ at different concentrations of 5mC DNA. b Plot of the intensity at 696 $\mathrm{cm}^{-1}$ with respect to the concentration of 5mC DNA. Insert shows linear dependence of the intensity in the logarithmic scale of DNA concentration over the range of $5 \times 10^{-3}-5 \mathrm{ng} \mu \mathrm{L}^{-1}$. Error bars show the standard deviation of a least five measurements. c SERS spectrum of $2 \mu \mathrm{mol} \mathrm{L}^{-1} \mathrm{SG}$ with different input methylation level in the mixtures of $5 \mathrm{mC}$ and unmethylated DNA. d Correlation of the measured and the actual input methylation levels in artificial mixtures of methylated and unmethylated DNA. Error bars show standard deviation from at least five replications.

\subsection{SERS detection of $5 \mathrm{hmC}$ and $5 \mathrm{caC}$}

After demonstrating the detection of 5mC DNA, next we extended our methods to detect its oxidation derivatives $5 \mathrm{hmC}$ and $5 \mathrm{caC}$. Their specific targeting antibodies were functionalized onto the AuNPs using a procedure similar to $5 \mathrm{mC}$ detection. As shown in Fig. 3, excellent performance was also obtained. By choosing $696 \mathrm{~cm}^{-1}$ as the characteristic peak for DNA quantification, the LOD for $5 \mathrm{hmC}$ was $\sim 0.2 \pm 0.05 \mathrm{pg} \mu \mathrm{L}^{-1}\left(\sim 1.8 \mathrm{pmol} \mathrm{L}^{-1}\right)$, a linear range between $0.5 \mathrm{pg} \mu \mathrm{L}^{-1}$ and 1 ng $\mu \mathrm{L}^{-1}$ was obtained. For $5 \mathrm{caC}$, a linear range between $0.05 \mathrm{ng} \mu \mathrm{L}^{-1}$ and $5 \mathrm{ng} \mu \mathrm{L}^{-1}$ with a LOD of $\sim 1 \pm 0.1 \mathrm{pg} \mu \mathrm{L}^{-1}\left(\sim 9.0 \mathrm{pmol} \mathrm{L}{ }^{-1}\right)$ was realized. Our results were better than the LOD possible with other detection methods.(Chowdhury et al. 2014; Hu and Zhang 2012; Wang et al. 2015a) More importantly, our approach requires very less input DNA $(1 \mu \mathrm{L}$, namely the input methylated DNA could be as low as $200 \mathrm{pg}$ ) and detection could be accomplished within 60 minutes, which would be very useful in clinical applications, and also as a pre-treatment for cancer research before gene specific studies with methods such as MSP.
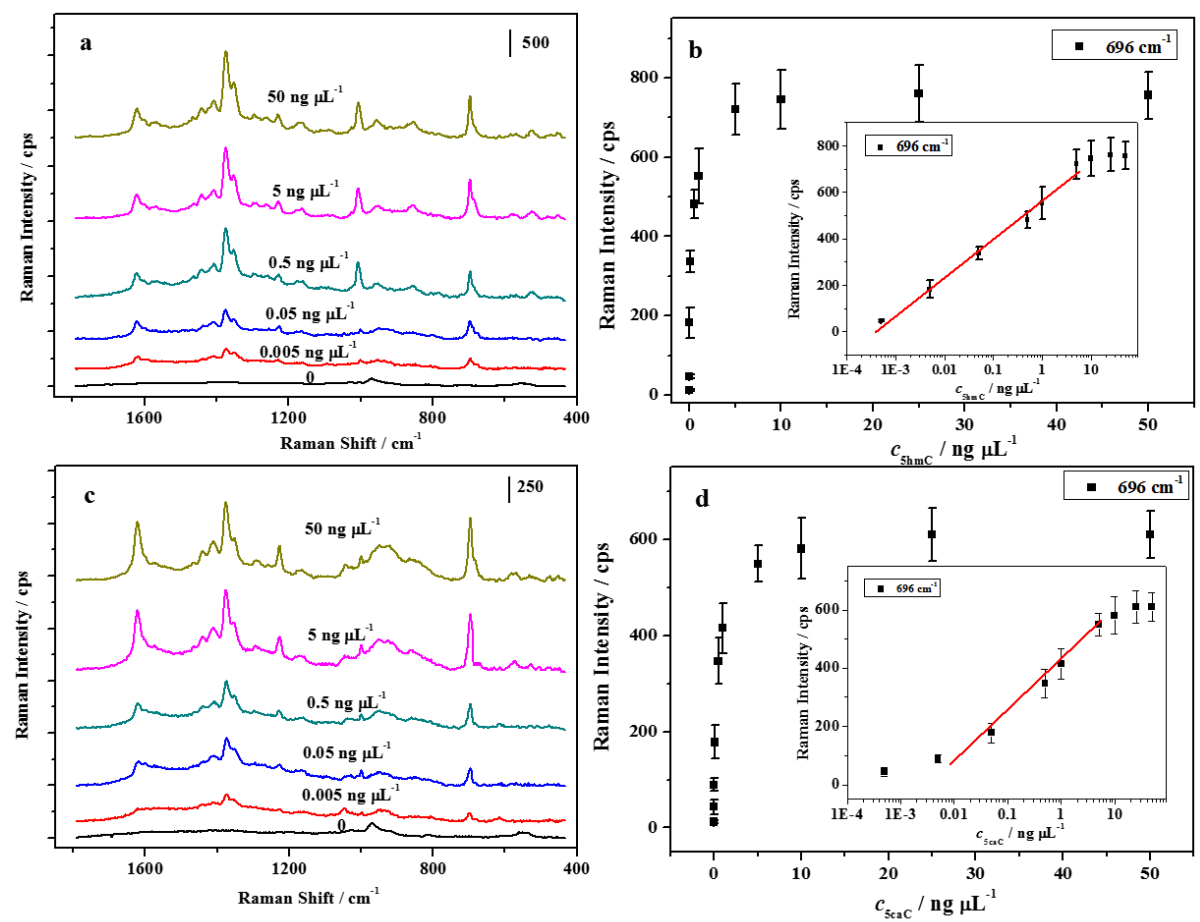

Fig. 3 a SERS spectrum of $2 \mu \mathrm{mol} \mathrm{L}{ }^{-1}$ SG for different concentrations of 5hmC DNA. b Plot of the intensity at 696 
$\mathrm{cm}^{-1}$ with respect to the concentration of 5hmC DNA. The insert shows linear dependence of the intensity with respect to the logarithm of DNA concentration over the range $0.5 \mathrm{pg} \mu \mathrm{L}^{-1}$ to $1 \mathrm{ng} \mu \mathrm{L}^{-1}$. Error bars show the standard deviation of a least five detections. c SERS spectrum of $2 \mu \mathrm{mol} \mathrm{L}^{-1} \mathrm{SG}$ at different concentration of $5 \mathrm{caC}$ DNA. d Plot of the intensity at $696 \mathrm{~cm}^{-1}$ with respect to the concentration of $5 \mathrm{caC}$ DNA. The insert shows a linear dependence of the intensity with respect to the logarithm of DNA concentration over the range of $0.05 \mathrm{ng} \mu \mathrm{L}^{-1}$ to 5 ng $\mu \mathrm{L}^{-1}$. Error bars show the standard deviation from five replications.

\subsection{Reproducibility and selectivity of the proposed method}

Excellent sensitivity is always an advantage in the SERS-based method, however, such a highly sensitive response to the local interaction between analytes and the substrate could also lead to poor reproducibility, which is one of the major weakness of the SERS approach.(Haynes et al. 2005) With our technique, much attention and efforts are expended in retaining signal reproducibility. First, uncontrollable movement or aggregation induced signal variation is prevented by homogenous self-assembly of AgNPs. The obtained Ag array not only provides excellent homogeneity, but also ample hot spots for SERS enhancement. Second, the direct interaction between $\mathrm{Ag}$ and $\mathrm{Au}$ captured DNA is prevented by a single layer graphene, the chemically induced signal alteration is also inhibited. Thus, two-dimensional graphene-Ag array based structures would provide excellent homogeneity. Experiments were performed to examine the reproducibility of our method to detect $5 \mathrm{mC}$ at $5 \mathrm{ng} \mu \mathrm{L}^{-1}$ (for a total of 5 replications). For each experiment, ten different measurements were obtained at random detection spots on the substrate. We also used five substrates from five batches of synthesis to evaluate the reproducibility between substrates from different batches, in total 50 spectrum were obtained. As shown in Fig. S6a, good homogeneity was obtained from ten different measurement positions. The intensity at $696 \mathrm{~cm}^{-1}$ was provided in Fig. S6b, an RSD of $\sim 8.21 \%$ was obtained, confirming the reproducibility of our approach. For comparison, we also performed a similar experiment with the graphene-Ag array as substrate, as shown in Fig. S6c, without laser shocking, the intensity RSD was about $12.72 \%$, because of the uneven interaction between Ag NPs and graphene layer.

Furthermore, due to the coexistence of these biomarkers in genomic DNA, it is critical to eliminate cross-reactivity and selectivity, which is an important factor in evaluating the analytical sensitivity for methylation detection. Multiple cross-reactivity tests were performed to examine the selectivity of this method, and the results demonstrated high specificity without significant cross-reactivity when three methylation related marks were detected (illustrated in Fig. 4). 


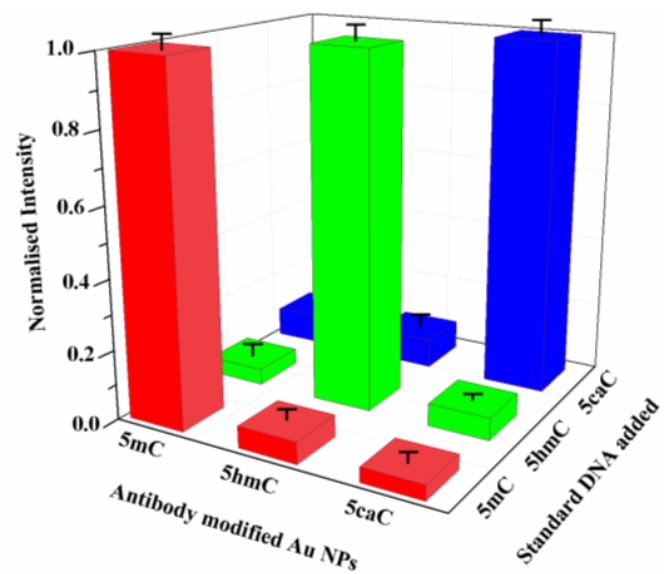

Fig. 4 Multiple cross-reactivity test to determine the selectivity of the proposed method. The $\mathrm{x}$-axis represents the antibody used for AuNPs modification (anti-5mC, $5 \mathrm{hmC}$, or $5 \mathrm{caC}$ ), Y-axis denotes the standard DNA used in each test and Z-axis denotes the corresponding SERS intensity at $696 \mathrm{~cm}^{-1}$ (the intensity was normalized to the strongest intensity in each type of AuNPs). The RSD for at least five detections were below 10\%, as shown by error bars.

\subsection{Reusability of the substrate}

The laser wrapped graphene onto the Ag array has several advantages, besides the fact that the enhancement provided by graphene wrapped Ag array in the form of a tight cover on the Ag array results in a low LOD and reusability of the substrate. Since there is no specific chemical interaction between single layer graphene to the target DNA that are captured on the AuNPs, it is easy to wash all of the AuNPs and DNA away that are dipped onto the graphene-Ag array, thus the substrate could be recycled. As shown in Fig. S7, after washing with water and ethanol once, the observed SERS response was significantly reduced, and after washed three times, no obvious signal from the probe was observed, implying that nearly all of the AuNPs with target DNA were washed away. The washed substrate was then applied into another round of detection. As confirmed in Fig. 5, we further used the substrate for other four cycles and obtained satisfactory results, exhibiting excellent reusability of the graphene wrapped Ag array substrate.

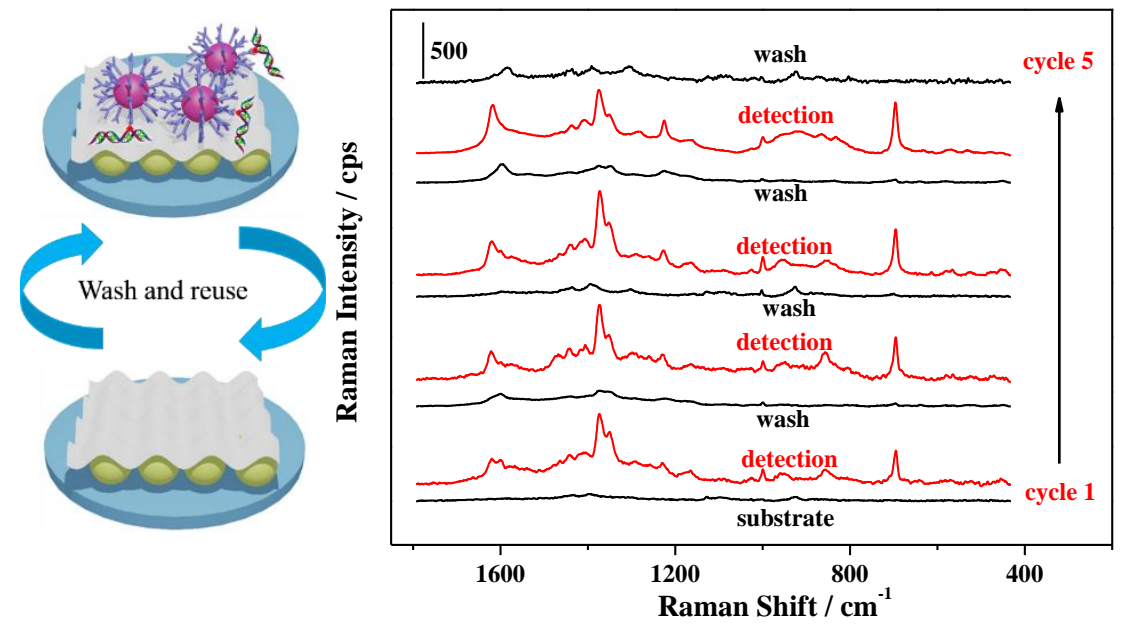

Fig. 5 SERS spectrum of SG marked 5mC-DNA dipped in graphene-Ag array before and after washing with water 
and ethanol.

\section{Conclusion}

Laser wrapped graphene-Ag array was proposed as a new substrate for sensitive detection of methylated DNA and its oxidation derivatives by SERS. By employing antibody affinity based enrichment strategy, trace target DNA was captured and labeled with the SERS tag, SG, whose signal was further enhanced by the graphene wrapped Ag array substrate. Such a strategy was successfully applied for trace detection of $5 \mathrm{mC}$ and two types of oxidation derivatives (5hmC, and $5 \mathrm{caC})$ with excellent reproducibility and selectivity. A detection limit as low as $0.2 \mathrm{pg} \mu \mathrm{L}^{-1}(1.8$ pmol L $\mathrm{L}^{-1}$ ) was achieved with ability to identify target DNA from DNA mixture in a ratio as low as $0.1 \%$, which was much better than the ELISA approaches and single base extension reaction based methods. The detection could be accomplished in less than 60 minutes with good accuracy (without PCR amplification or conversion steps that might induce false signal) was successfully applied for the detection of $5 \mathrm{mC}$ in real cell samples. The proposed method shows excellent prospect for clinical application especially when it is combined with portable instruments. Information on global methylation will provide the basis to indicate gene-specific studies. The developed structure of uniformly assembled Ag array wrapped with graphene facilitated the transfer of electromagnetic field for signal enhancement, and was also reusable for several cycles, demonstrating its practical relevance. The unique SERS sensor design could also be applied in the analysis of other important biomarkers. The detection strategy, by making use of affinity capture and signal amplification by graphene-Ag array also holds promise in the detection of other important biological targets, such as histone modifications.

\section{Supplementary Material.}

The detailed experimental steps and parameters and Figures S1-S7 are included in the Supplementary Material.

\section{Corresponding Authors}

Professor Lihua Zhu, College of Chemistry and Chemical Engineering, Huazhong University of Science and Technology, Wuhan 430074, P. R. China. E-mail: lhzhu63@mail.hust.edu.cn.

Professor Joseph Irudayaraj, Department of Agriculture \& Biological Engineering, Bindley Bioscience Center, Purdue Center for Cancer Research, Purdue University, West Lafayette, Indiana 47907, USA. E-mail: josephi@ purdue.edu

\section{Acknowledgment}


This work was supported by the W.M. Keck Foundation grant. Partial support from the USDA-Purdue Center for Food Safety Engineering is appreciated. We thank Xiaolei Wang for helping in FDTD simulation. The author Ouyang Lei is thankful for the funding from Chinese Scholarship Council (CSC).

\section{Reference}

Bailey, V.J., Easwaran, H., Zhang, Y., Griffiths, E., Belinsky, S.A., Herman, J.G., Baylin, S.B., Carraway, H.E., Wang, T.-H., 2009. MS-qFRET: a quantum dot-based method for analysis of DNA methylation. Genome research 19(8), 1455-1461.

Baylin, S.B., Jones, P.A., 2011. A decade of exploring the cancer epigenome-biological and translational implications. Nature Reviews Cancer 11(10), 726-734.

Cao, A., Zhang, C.-y., 2012. Sensitive and label-free DNA methylation detection by ligation-mediated hyperbranched rolling circle amplification. Analytical chemistry 84(14), 6199-6205.

Chowdhury, B., Cho, I.-H., Hahn, N., Irudayaraj, J., 2014. Quantification of 5-methylcytosine, 5-hydroxymethylcytosine and 5-carboxylcytosine from the blood of cancer patients by an enzyme-based immunoassay. Analytica chimica acta 852, 212-217.

Chowdhury, B., Seetharam, A., Wang, Z., Liu, Y., Lossie, A.C., Thimmapuram, J., Irudayaraj, J., 2016. A Study of Alterations in DNA Epigenetic Modifications (5mC and 5hmC) and Gene Expression Influenced by Simulated Microgravity in Human Lymphoblastoid Cells. PloS one 11(1), e0147514.

Du, Y., Zhao, Y., Qu, Y., Chen, C.-H., Chen, C.-M., Chuang, C.-H., Zhu, Y., 2014. Enhanced light-matter interaction of graphene-gold nanoparticle hybrid films for high-performance SERS detection. Journal of Materials Chemistry C 2(23), 4683-4691.

Feinberg, A.P., Vogelstein, B., 1983. Hypomethylation distinguishes genes of some human cancers from their normal counterparts. Nature 301(5895), 89-92.

Feng, F., Wang, H., Han, L., Wang, S., 2008. Fluorescent conjugated polyelectrolyte as an indicator for convenient detection of DNA methylation. Journal of the American Chemical Society 130(34), 11338-11343.

Frommer, M., McDonald, L.E., Millar, D.S., Collis, C.M., Watt, F., Grigg, G.W., Molloy, P.L., Paul, C.L., 1992. A genomic sequencing protocol that yields a positive display of 5-methylcytosine residues in individual DNA strands. Proceedings of the National Academy of Sciences 89(5), 1827-1831.

Gama-Sosa, M.A., Slagel, V.A., Trewyn, R.W., Oxenhandler, R., Kuo, K.C., Gehrke, C.W., Ehrlich, M., 1983. The 5-methylcytosine content of DNA from human tumors. Nucleic acids research 11(19), 6883-6894.

Gao, H., Hu, Y., Xuan, Y., Li, J., Yang, Y., Martinez, R.V., Li, C., Luo, J., Qi, M., Cheng, G.J., 2014. Large-scale nanoshaping of ultrasmooth 3D crystalline metallic structures. Science 346(6215), 1352-1356.

Garcia-Leis, A., Garcia-Ramos, J.V., Sanchez-Cortes, S., 2013. Silver nanostars with high SERS performance. The Journal of Physical Chemistry C 117(15), 7791-7795.

Haynes, C.L., McFarland, A.D., Duyne, R.P.V., 2005. Surface-enhanced Raman spectroscopy. Analytical Chemistry 77(17), 338 A-346 A.

Herman, J.G., Graff, J.R., Myöhänen, S., Nelkin, B.D., Baylin, S.B., 1996. Methylation-specific PCR: a novel PCR assay for methylation status of CpG islands. Proceedings of the National Academy of Sciences 93(18), 9821-9826.

Hu, J., Zhang, C.-y., 2012. Single base extension reaction-based surface enhanced Raman spectroscopy 
for DNA methylation assay. Biosensors and Bioelectronics 31(1), 451-457.

Hu, Y., Lee, S., Kumar, P., Nian, Q., Wang, W., Irudayaraj, J., Cheng, G.J., 2015. Water flattens graphene wrinkles: laser shock wrapping of graphene onto substrate-supported crystalline plasmonic nanoparticle arrays. Nanoscale 7(47), 19885-19893.

Huang, W., Qi, C.-B., Lv, S.-W., Xie, M., Feng, Y.-Q., Huang, W.-H., Yuan, B.-F., 2016. Determination of DNA and RNA Methylation in Circulating Tumor Cells by Mass Spectrometry. Analytical chemistry 88(2), 1378-1384.

Ito, S., Shen, L., Dai, Q., Wu, S.C., Collins, L.B., Swenberg, J.A., He, C., Zhang, Y., 2011. Tet proteins can convert 5-methylcytosine to 5-formylcytosine and 5-carboxylcytosine. Science 333(6047), 1300-1303.

Jones, P.A., Taylor, S.M., 1980. Cellular differentiation, cytidine analogs and DNA methylation. Cell 20(1), 85-93.

Kato, D., Goto, K., Fujii, S.-i., Takatsu, A., Hirono, S., Niwa, O., 2011. Electrochemical DNA methylation detection for enzymatically digested CpG oligonucleotides. Analytical chemistry 83(20), 7595-7599.

Laird, P.W., 2010. Principles and challenges of genome-wide DNA methylation analysis. Nature Reviews Genetics 11(3), 191-203.

Le, T., Kim, K.-P., Fan, G., Faull, K.F., 2011. A sensitive mass spectrometry method for simultaneous quantification of DNA methylation and hydroxymethylation levels in biological samples. Analytical biochemistry 412(2), 203-209.

Lee, S., Kumar, P., Hu, Y., Cheng, G.J., Irudayaraj, J., 2015. Graphene laminated gold bipyramids as sensitive detection platforms for antibiotic molecules. Chemical Communications 51(85), 15494-15497.

Li, C., Sun, L., Sun, Y., Teranishi, T., 2013. One-Pot controllable synthesis of Au@ Ag heterogeneous nanorods with highly tunable plasmonic absorption. Chemistry of Materials 25(13), 2580-2590.

Oda, M., Glass, J.L., Thompson, R.F., Mo, Y., Olivier, E.N., Figueroa, M.E., Selzer, R.R., Richmond, T.A., Zhang, X., Dannenberg, L., 2009. High-resolution genome-wide cytosine methylation profiling with simultaneous copy number analysis and optimization for limited cell numbers. Nucleic acids research 37(12), 3829-3839.

Ouyang, L., Zhu, L., Ruan, Y., Tang, H., 2015. Preparation of a native $\beta$-cyclodextrin modified plasmonic hydrogel substrate and its use as a surface-enhanced Raman scattering scaffold for antibiotics identification. Journal of Materials Chemistry C 3(29), 7575-7582.

Shames, D.S., Minna, J.D., Gazdar, A.F., 2007. Methods for detecting DNA methylation in tumors: from bench to bedside. Cancer letters 251(2), 187-198.

Shanmuganathan, R., Basheer, N.B., Amirthalingam, L., Muthukumar, H., Kaliaperumal, R., Shanmugam, K., 2013. Conventional and nanotechniques for DNA methylation profiling. The Journal of Molecular Diagnostics 15(1), 17-26.

Singer-Sam, J., LeBon, J.M., Tanguay, R.L., Riggs, A.D., 1990. A quantitative HpaII-PCR assay to measure methylation of DNA from a small number of cells. Nucleic acids research 18(3), 687.

Song, C.-X., He, C., 2012. Balance of DNA methylation and demethylation in cancer development. Genome biology 13(10), 1.

Song, C.-X., He, C., 2013. Potential functional roles of DNA demethylation intermediates. Trends in biochemical sciences 38(10), 480-484.

Stiles, P.L., Dieringer, J.A., Shah, N.C., Van Duyne, R.P., 2008. Surface-enhanced Raman spectroscopy. 
Annu. Rev. Anal. Chem. 1, 601-626.

Sun, L., Yu, C., Irudayaraj, J., 2007. Surface-enhanced Raman scattering based nonfluorescent probe for multiplex DNA detection. Analytical chemistry 79(11), 3981-3988.

Sun, L., Yu, C., Irudayaraj, J., 2008. Raman multiplexers for alternative gene splicing. Analytical chemistry 80(9), 3342-3349.

Taleat, Z., Mathwig, K., Sudhölter, E.J., Rassaei, L., 2015. Detection strategies for methylated and hypermethylated DNA. TrAC Trends in Analytical Chemistry 66, 80-89.

Timp, W., Feinberg, A.P., 2013. Cancer as a dysregulated epigenome allowing cellular growth advantage at the expense of the host. Nature Reviews Cancer 13(7), 497-510.

Umezu, T., Ohyashiki, K., Ohyashiki, J.H., 2011. Detection method for quantifying global DNA methylation by fluorescence correlation spectroscopy. Analytical biochemistry 415(2), 145-150.

Wang, M., Xu, Z., Chen, L., Yin, H., Ai, S., 2012a. Electrochemical immunosensing platform for DNA methyltransferase activity analysis and inhibitor screening. Analytical chemistry 84(21), 9072-9078.

Wang, P., Chen, H., Tian, J., Dai, Z., Zou, X., 2013. Electrochemical evaluation of DNA methylation level based on the stoichiometric relationship between purine and pyrimidine bases. Biosensors and Bioelectronics 45, 34-39.

Wang, X., Cui, M., Zhou, H., Zhang, S., 2015a. DNA-hybrid-gated functional mesoporous silica for sensitive DNA methyltransferase SERS detection. Chemical Communications 51(73), 13983-13985.

Wang, X., Cui, Y., Irudayaraj, J., 2015b. Single-Cell Quantification of Cytosine Modifications by Hyperspectral Dark-Field Imaging. ACS nano 9(12), 11924-11932.

Wang, Y., Wee, E.J., Trau, M., 2016. Accurate and sensitive total genomic DNA methylation analysis from sub-nanogram input with embedded SERS nanotags. Chemical Communications 52(17), 3560-3563.

Wang, Y., Zhang, C.-H., Tang, L.-J., Jiang, J.-H., 2012b. Enzymatic control of plasmonic coupling and surface enhanced Raman scattering transduction for sensitive detection of DNA demethylation. Analytical chemistry 84(20), 8602-8606.

Wu, Y., Zhang, B., Guo, L.-H., 2013. Label-free and selective photoelectrochemical detection of chemical DNA methylation damage using DNA repair enzymes. Analytical chemistry 85(14), 6908-6914.

Xu, S., Jiang, S., Wang, J., Wei, J., Yue, W., Ma, Y., 2016. Graphene isolated Au nanoparticle arrays with high reproducibility for high-performance surface-enhanced Raman scattering. Sensors and Actuators B: Chemical 222, 1175-1183.

Yin, H., Zhou, Y., Xu, Z., Chen, L., Zhang, D., Ai, S., 2013. An electrochemical assay for DNA methylation, methyltransferase activity and inhibitor screening based on methyl binding domain protein. Biosensors and Bioelectronics 41, 492-497.

Yu, Y., Blair, S., Gillespie, D., Jensen, R., Myszka, D., Badran, A.H., Ghosh, I., Chagovetz, A., 2010. Direct DNA methylation profiling using methyl binding domain proteins. Analytical chemistry 82(12), 5012-5019.

Zhang, H., Li, M., Fan, M., Gu, J., Wu, P., Cai, C., 2014. Electrochemiluminescence signal amplification combined with a conformation-switched hairpin DNA probe for determining the methylation level and position in the Hsp53 tumor suppressor gene. Chemical Communications 50(22), 2932-2934.

Zhou, H., Lin, S., Nie, Y., Yang, D., Wang, Q., Chen, W., Huang, N., Jiang, Z., Chen, S., 2015a. Surface-enhanced resonance Raman scattering (SERRS) simulates PCR for sensitive DNA detection. 
Analyst 140(22), 7518-7521.

Zhou, W., Gao, X., Liu, D., Chen, X., 2015b. Gold nanoparticles for in vitro diagnostics. Chemical reviews 115(19), 10575-10636.

Zhou, Z., Cho, I.-H., Shan, Z., Irudayaraj, J., 2015c. Cross-platform detection of epigenetic modifications from extracted chromatin in leucocytes from blood. Analytical Chemistry Research 4, $39-44$. 